\title{
Analysis of the Advantages of Wiltse Approach Ligamentotaxis Method for Thoracolumbar Fractures
}

\author{
Baixing Wei, Taipu Liu, Hongfei Li, Xipeng Chen and Han Wu \\ Department of Orthopedics, China Japan Union Hospital of Jilin University, China
}

\begin{abstract}
Objective: The Wiltse approach ligamentotaxis (WALT) is a combination of paravertebral muscle natural gap access and posterior longitudinal ligament tension, which can achieve good fracture repositioning and indirect spinal canal decompression, thus avoiding the disadvantages of traditional posterior direct decompression approach. This paper provides a detailed explanation and analysis of WALT through clinical cases.
\end{abstract}

Methods: 103 patients with thoracolumbar vertebral fractures classified as AO.A surgically treated with the spinal screw bar system from 2017.01 to 2020.06 were included, of whom 52 were performed with indirect decompression and repositioning of the spinal canal by the WALT method (hereafter referred to as the Wiltse group) and 51 were performed with direct decompression and repositioning of the spinal canal by traditional posterior direct decompression approach (hereafter referred to as the traditional group), and the results were compared by operating time, bleeding, incision length, preoperative and postoperative changes in relative vertebral body height and Cobb angle, preoperative and postoperative Visual analogue scores (VAS) and Frankel scores, postoperative Oswestry disability index (ODI), postoperative drainage flow, postoperative time to remove drainage devices, and postoperative hospitalization days to compare the surgical results of fractures in the two groups.

Results: The Wiltse group had an operative time of 76.5(65-110) min, bleeding volume of 90(50-180) ml, incision length of $9.4(7.8-11.3) \mathrm{cm}$, postoperative drainage of $97.4 \pm 34.7 \mathrm{ml}$, postoperative drainage removal time of 2(1-4) days, postoperative hospitalization of 4(3-6) days, VAS score of $0(0-1)$ for 1 year postoperatively and ODI score 5(2-8) for 1 year postoperatively. In the traditional approach group, the operative time was 120(90-145) min, bleeding volume was 190(140-300) ml, incision length was $16.5(14.0-18.5) \mathrm{cm}$, postoperative drainage flow was $181.7 \pm 41.0 \mathrm{ml}$, postoperative drainage removal time was $4(2-6)$ days, and postoperative hospital stay was 6(3-8) days. days, VAS score 1(1-2) 1 year postoperatively, ODI score 6(3-10) 1 year postoperatively. The differences were significant in both groups $(P<0.05)$. There were no statistically significant differences in changes of vertebral height, Cobb Angle and Frankel score 1 year postoperatively $(P>0.05)$.

Conclusion: The Wiltse approach reveals the vertebral pedicle and vertebral body through the paravertebral muscle gap separation without stripping the paravertebral muscle from the lamina and without removing and destroying bony structures such as the lamina, thus reducing the operative time and bleeding and avoiding the medically induced injuries caused by traditional surgery. This approach combined with the ligament tension repositioning method for indirect decompression and repositioning of the spinal screw bar system can achieve the same effectiveness as traditional surgery, which is a minimally invasive surgical method that can replace most traditional surgeries and is worth studying and promoting.

\section{Keywords}

Thoracolumbar fracture, Wiltse approach, Ligamentous tension repositioning

\section{Abbreviations}

WALT: The Wiltse Approach Ligamentotaxis; VAS: Visual Analogue Scores; ODI: Oswestry Disability Index; MRI: Magnetic Resonance Imaging; CT: Computed Tomography

\section{Introduction}

The thoracolumbar segment of the spine is T10-L2, which is the conversion point of the thoracic and lumbar vertebrae, this area of the lumbar vertebrae relative to the thoracic vertebrae mobility, and this junction of the spine itself is more active, carrying a large load, but also for the lumbar is major muscle and paraspinal muscle protection is weakened area, and therefore most often injured [1]. The thoracolumbar spinal canal is also a region of spinal cord, conus, cauda equina,
*Corresponding author: Han Wu, MD, PhD, Department of Orthopedics, China-Japan Union Hospital of Jilin University, Jilin University, No. 126 Xi'antai Avenue, Changchun City, Jilin Province, 130000, China, Tel: +13-514-462-233

Accepted: September 24, 2021

Published online: September 26, 2021

Citation: Wei B, Liu T, Li H, et al. (2021) Analysis of the Advantages of Wiltse Approach Ligamentotaxis Method for Thoracolumbar Fractures. J Orthop Surg Tech 4(2):364-374 
and nerve root migration, and the space in the spinal canal is narrow, so the thoracolumbar injury is likely to cause spinal cord nerve compression, injury, and subsequent sensory or motor impairment of the lower extremities or urinary and fecal dysfunction [1]. In the case of thoracolumbar fractures, conservative treatment requires prolonged bed rest and carries the risk of secondary spinal nerve injury and posterior convexity deformity. Patients treated conservatively have a poor quality of life, are unable to walk, suffer from chronic back pain, and are at risk for complications such as bone loss and pressure sores due to prolonged bed rest. In addition, the cost of care is high [2]. In general, thoracolumbar fractures are usually treated surgically with the implantation of a spinal screw rod system to obtain repositioning and fixation of the fracture. Traditional posterior direct decompression approach involves stripping the paravertebral muscles from the lamina, exposing the spinous process, the lamina, and the small articular processes, and then repositioning and fixing the fracture with spinal screw rod system; for larger bone masses occupying the vertebral canal, direct decompression by laminectomy is required [1]. There are drawbacks to traditional surgery, such as intractable postoperative low back pain, which is often associated with loss of innervation, ischemia, atrophy, disuse, and necrosis of the paravertebral muscles caused by paravertebral muscle stripping [3]. In addition, the long operation time and high bleeding are also the disadvantages of direct decompression in the traditional operation.

The posterior longitudinal ligament plays a role in maintaining the stability of the middle column and preventing displacement of the intervertebral discs and vertebral bodies [4]. In recent years, for some cases of compression fractures and burst fractures in which the posterior longitudinal ligament is intact, we have used the Wiltse approach combined with the posterior longitudinal ligament tension repositioning method, using the longitudinal force generated by the nail bar system to brace the intact posterior longitudinal ligament to produce transverse tension out of the spinal canal to reposition the fracture, and achieved good results. This avoids stripping of the paravertebral muscle and direct removal of the laminae for decompression, preventing loss of innervation, ischemia, necrosis, and loss of use of the paravertebral muscle, and reduces the risk of postoperative muscle hematoma to a certain extent.

And how to describe the injury of posterior longitudinal ligament, we took the approach of classifying it by AO typing [5]. $A O$ typing is divided into three types in $A, B$ and $C$. Among type $A, A 0$ is a fracture involving the spinous process or transverse process, which does not require surgical treatment, while the rest of type $A$ have in common that the posterior longitudinal ligament complex is intact and the trauma does not injure the posterior longitudinal ligament, only some compression or burst fractures involving the upper and lower cartilage endplates. Type $B$ fractures contain chance fractures and hyperextension fractures that destroy the anterior tension band damage. Type B fractures have a posterior longitudinal ligament complex and damage to the posterior longitudinal ligament. Type $\mathrm{C}$ is a fracture dislocation, so the typing is not meticulous because the fracture pattern varies. In this paper, we discuss and analyze the advantages of the wiltse approach ligament tension repositioning method for the treatment of AO.A type of thoracolumbar vertebral fractures except type A0 through clinical cases.

\section{Materials and Methods}

\section{Inclusion and exclusion criteria}

Inclusion criteria: Patients with thoracolumbar fractures surgically treated with an internal spinal fixation system from January 2017 to June 2020 were retrospectively selected. Among the qualifying criteria for inclusion in the study were patients with single-segment thoracolumbar segment fractures with an AO.A staging other than AO.0, [5] where the posterior longitudinal ligament and posterior longitudinal ligament complex were undamaged in patients with fractures of this staging. The surgical treatment was internal fixation with two pedicle screws in each of the injured vertebrae and the upper and lower vertebrae.

Exclusion criteria: Morethanonesegmentalthoracolumbar fracture; AO.B and AO.C thoracolumbar fractures; or patients with combined ankylosing spondylitis, osteoporosis, scoliosis, and deformities of the thoracolumbar spine. Patients with multiple injuries who underwent other surgical procedures during hospitalization or who had combined injuries to other parts of the body were also excluded from this study because this may affect the length of postoperative hospitalization and the complexity of postoperative supportive and nursing care, and may affect the ratings of each score.

The final number of patients included in the study was 103. Of these, 52 cases were indirectly decompressionreduced via the WALT method (hereafter referred to as the Wiltse group) and 51 cases were decompression-reduced by the traditional posterior direct decompression approach (hereafter referred to as the traditional group). Various data of both groups were counted, and the age, gender, vertebral fracture segment, operation time, bleeding, incision length, change in the sagittal Cobb angle of the relative height of the fractured vertebrae before and after surgery (calculated as a percentage due to the difference in lengths obtained from $X$-ray and CT measurements; the imaging data of all cases were measured three times and averaged as the final results), postoperative VAS score, pre-and postoperative VAS score (where postoperative ODI and VAS scores were evaluated once on postoperative day 3 , once at postoperative month 6 , and once at postoperative month 12), preoperative and postoperative Frankel scores at 12 months, postoperative drainage flow, postoperative drainage removal time (drainage was removed on the day when the drainage was less than $50 \mathrm{ml}$ ), and postoperative hospitalization days. The data obtained were also analyzed statistically using SPSS 13.0 as a way to compare the degree of surgical repositioning of fractures and the treatment effect between the two groups and to analyze the advantages of each of the two procedures.

\section{Surgical method}

Posterior median approach for direct decompression 
(conventional group): After anesthesia takes effect, the patient is placed in prone position, and the C-arm X-ray machine is used to locate the pedicle where the nail must be placed. A median skin incision is made, a posterior median longitudinal incision is made with the injured vertebral arch as the center, the skin is incised layer by layer, the subcutaneous tissue is removed to the supraspinous ligament, the paravertebral muscles on both sides are stripped under the periosteum to the outer edge of the articular eminence, the vertebral plates and articular eminence on both sides are exposed, holes are drilled in the direction of the injured upper and lower adjacent vertebral arch, a positioning pin is placed, the C-arm fluoroscopy is adjusted to the ideal position, and the arch nail is placed respectively. If there is an occupied fracture bone in the vertebral canal, laminectomy is performed to decompress and push the top or remove the free fracture bone to reset the fracture. The upper connecting rod is fixed with a top wire. Layer by layer suture dressing was applied.

WALT (Wiltse group): After general anesthesia is in effect, the patient is placed in prone position and the C-arm X-ray machine fluoroscopy is used to locate the pedicle where the nail must be placed. A posterior median longitudinal incision was made with the injured vertebral arch as the center, and a subcutaneous separation was made to both sides of the longest muscle gap between the multifidus muscle and the longest muscle gap, then a blunt separation was made along this gap to reveal both sides of the synovial joint, and holes were drilled in the direction of the pinned vertebral arch respectively, a positioning pin was placed, and the C-arm fluoroscopy was adjusted to the ideal position, and the pinned vertebral arch, connecting rod and top wire were placed, and the pinned vertebral arch was propped up on both sides for about 1.0-1.5 cm and the top wire was fixed. The C-arm X-ray machine was used to observe the recovery of vertebral body height and the flatness of the posterior edge of the vertebral body until the fracture was well repositioned, and the incision was closed and bandaged layer by layer (Figure 1).

Both groups were operated by the same senior physician as the primary surgeon, and the surgery was completed within 48 hours of admission.

\section{Statistical analysis}

The length of surgical incision, operating time, intraoperative bleeding, preoperative and postoperative changes in the Cobb angle of the injured vertebrae, and percentage recovery of vertebral body height were calculated for both groups (relative height of the anterior margin of the injured vertebrae $=$ the mean of the actual height of the anterior margin of the injured vertebrae/the sum of the height of the anterior margins of the adjacent upper and lower vertebrae $\times 100 \%$. The angle of intersection of the two vertical lines is the sagittal Cobb angle), preoperative and postoperative ODI scores, VAS scores, postoperative hospitalization days, postoperative drainage removal time, and postoperative drainage flow were made on the lateral film (All imaging data were averaged for three separate measurements). These indices were compared and statistically analyzed. SPSS 25.0 was used for statistical analysis, and measurement data conforming to normal distribution were expressed as mean \pm standard deviation

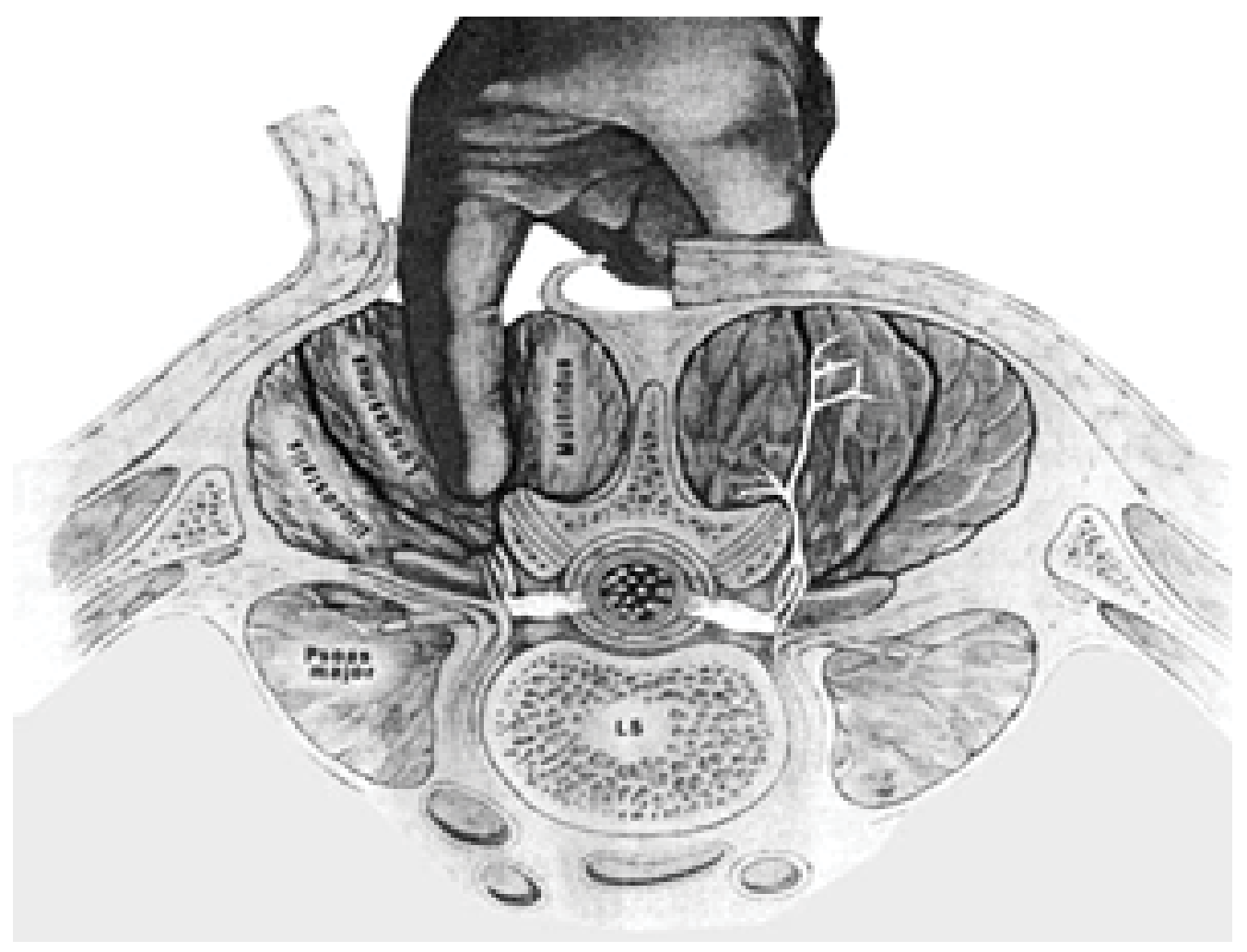

Figure 1: The picture shows a finger probe of the vertebral body and pedicle. 
Citation: Wei B, Liu T, Li H, et al. (2021) Analysis of the Advantages of Wiltse Approach Ligamentotaxis Method for Thoracolumbar Fractures. J Orthop Surg Tech 4(2):364-374

and compared using t-test (two-sided), while measurement data not conforming to normal distribution were expressed as median (min-max) and compared between groups using non-parametric test. Count data were expressed as numbers, and the $x$-test was used for comparison between groups, and $P<0.05$ was considered significant.

\section{Results}

A total of 103 patients were included in the study, $51 \pm 8$ in the Wiltse group, age 38 males, 14 females, 2 T10 fractures,
4 T11 fractures, 11 T12 fractures, 24 L1 fractures, 10 L2 fractures; 2 in the traditional group, age $46 \pm 11,32$ males, 19 females, 2 T10 fractures, 5 T11 fractures, 11 T12 fractures There were 2 T10 fractures, 5 T11 fractures, 11 T12 fractures, $24 \mathrm{~L} 1$ fractures, and $10 \mathrm{~L} 2$ fractures. In the traditional group, 15 were $A 1,2$ were $A 2,29$ were $A 3$, and 5 were $A 4$. In the Wiltse group, 17 were $A 1,1$ were $A 2,26$ were $A 3$, and 8 were A4. Baseline analysis of the two groups showed no statistically significant differences in gender, age, surgical segment, and AO type (Table 1$)$.

Table 1: Baseline information table.

\begin{tabular}{|c|c|c|c|}
\hline & Traditional group & Wiltse group & $P$ value \\
\hline & $(N=51)$ & $(N=52)$ & \\
\hline Gender & & & 0.296 \\
\hline Male & 32 & 38 & \\
\hline Female & 19 & 14 & \\
\hline Age & $46 \pm 11$ & $51 \pm 8$ & 0.017 \\
\hline Injured vertebral & & & 0.988 \\
\hline T10 & 2 & 2 & \\
\hline T11 & 5 & 4 & \\
\hline T12 & 11 & 11 & \\
\hline L1 & 24 & 24 & \\
\hline L2 & 10 & 10 & \\
\hline AO type & & & 0.728 \\
\hline A1 & 15 & 17 & \\
\hline A2 & 2 & 1 & \\
\hline A3 & 29 & 26 & \\
\hline A4 & 5 & 8 & \\
\hline
\end{tabular}

Table 2: Comparison of the surgical results among Wiltse group and traditional group.

\begin{tabular}{|c|c|c|c|c|}
\hline & All & Wiltse group & Traditional group & $p$ value \\
\hline Surgery time & $95(65-145)$ & $76.5(65-110)$ & $120(90-145)$ & $<0.001$ \\
\hline Bleeding volume & $140(50-300)$ & $90(50-180)$ & $190(140-300)$ & $<0.001$ \\
\hline Incision length & $11.3(7.8-18.5)$ & $9.4(7.8-11.3)$ & $16.5(14.0-18.5)$ & $<0.001$ \\
\hline 3 days ODI (Yes) & $25.4 \pm 5.2$ & $21.6 \pm 4.0$ & $29.3 \pm 2.8$ & $<0.001$ \\
\hline 6 months ODI & $11(5-18)$ & $9(5-15)$ & $13(8-18)$ & $<0.001$ \\
\hline 12 months ODI & $6(2-10)$ & $5(2-8)$ & $6(3-10)$ & $<0.001$ \\
\hline Pre-operative VAS & $8(6-10)$ & $8(6-10)$ & $8(7-10)$ & 0.568 \\
\hline 3 days VAS & $3(2-5)$ & $2.6(2-4)$ & $3.8(2-5)$ & $<0.001$ \\
\hline 6 months VAS & $1(0-3)$ & $1(0-3)$ & $2(1-3)$ & $<0.001$ \\
\hline 12 months VAS & $1(0-2)$ & $0(0-1)$ & $1(1-2)$ & $<0.001$ \\
\hline Post-operative drainage (Yes) & $139.1 \pm 56.7$ & $97.4 \pm 34.7$ & $181.7 \pm 41.0$ & $<0.001$ \\
\hline Postoperative drainage removal & $3(1-6)$ & $2(1-4)$ & $4(2-6)$ & $<0.001$ \\
\hline Postoperative hospital stay & $5(3-8)$ & $4(3-6)$ & $6(3-8)$ & $<0.001$ \\
\hline
\end{tabular}


Citation: Wei B, Liu T, Li H, et al. (2021) Analysis of the Advantages of Wiltse Approach Ligamentotaxis Method for Thoracolumbar Fractures. J Orthop Surg Tech 4(2):364-374

In the Wiltse group, the operative time was 76.5(65110) min, bleeding volume was $90(50-180) \mathrm{ml}$, incision length was $9.4(7.8-11.3) \mathrm{cm}$, postoperative drainage was $97.4 \pm 34.7 \mathrm{ml}$, postoperative drainage removal time was 2(1-4) days, postoperative hospital stay was 4(3-6) days, postoperative VAS score at 3 days was 2.6(2-4), 6-month postoperative VAS score $1(0-3), 12$-month VAS score $0(0-$ 1), 3-day postoperative ODI score $21.6 \pm 4.0$, 6-month postoperative ODI score 9(5-15), 12-month postoperative ODI score 5(2-8); the traditional group had an operative time of $120(90-145) \mathrm{min}$, a bleeding volume of $190(140-300) \mathrm{ml}$, and an incision length $16.5(14.0-18.5) \mathrm{cm}$, postoperative drainage $181.7 \pm 41.0 \mathrm{ml}$, postoperative drainage removal time 4(2-6) days, postoperative hospitalization days 6(3-8) days, postoperative VAS score 3.8(2-5) days, postoperative VAS score 2(1-3) 6 months, postoperative VAS score 1(12) 12 months, postoperative ODI score 3 days $29.3 \pm 2.8$, 6-month postoperative ODI score 13(8-18), and 12-month postoperative ODI score 6(3-10). The differences between both groups were significant $(\mathrm{P}<0.001)$ (Table 2$)$. There was no significant difference in Cobb angle difference before and after the two approaches ( $P>0.05)$, and the difference in vertebral height recovery status was extremely insignificant; the Frankel score recovery after 12 months postoperatively was approximately the same in both groups (Table 3 and Table 4).

Note: All patients underwent the operation smoothly without adverse events such as failure of internal fixation implantation and vascular nerve injury, and no complications such as fracture or prolapse of the internal fixation occurred in all patients during the 12-month follow-up period. The accuracy of pedicle screw placement was $96.5 \%$ (301/312) in Wiltse group and $97.4 \%(298 / 306)$ in traditional group, with no significant difference between the two groups.

\section{Discussion}

The thoracolumbar segment of the spine is the region of the spine where stresses are concentrated during motion and rotation, and statistics show that thoracolumbar fractures account for approximately $67 \%$ of spinal fractures [6]. For compression or burst fractures of the thoracolumbar vertebral, the traditional surgical treatment is to peel off the paravertebral muscles, expose the vertebral plate and the upper and lower articular joints, and then perform internal fixation through a nail rod system. In the case of a large bone mass occupying the vertebral canal, laminectomy decompression and pushing of the top free fracture bone mass is required [1]. Although paravertebral muscle approach hand surgery was first applied by Wiltse [7] for procedures such as lumbar fusion, in recent years domestic and international studies have concluded that it can be widely used for a variety of lumbar spine surgery. Our preliminary study [8] showed that the transmaxillary and multifidus approaches can be used for the vast majority of

Table 3: Comparison of the radiographic results of the two groups.

\begin{tabular}{|c|c|c|c|c|}
\hline & All & Wiltse group & Traditional group & p value \\
\hline \multicolumn{5}{|l|}{ Cobb angle } \\
\hline Preoperative & $13.4 \pm 7.7$ & $13.7 \pm 7.6$ & $13.0 \pm 7.8$ & 0.601 \\
\hline Postoperative & $4.2 \pm 7.0$ & $4.6 \pm 3.9$ & $3.9 \pm 7.4$ & 0.628 \\
\hline Cobb angle difference & $9.4 \pm 4.5$ & $9.6 \pm 4.8$ & $9.3 \pm 4.2$ & 0.783 \\
\hline \multicolumn{5}{|l|}{ Height ratio } \\
\hline Preoperative & $0.68 \pm 0.12$ & $0.67 \pm 0.11$ & $0.71 \pm 0.12$ & 0.037 \\
\hline Postoperative & $0.93 \pm 0.06$ & $0.93 \pm 0.06$ & $0.93 \pm 0.06$ & 0.999 \\
\hline Ratio difference & $0.24 \pm 0.12$ & $0.27 \pm 0.11$ & $0.22 \pm 0.12$ & 0.037 \\
\hline
\end{tabular}

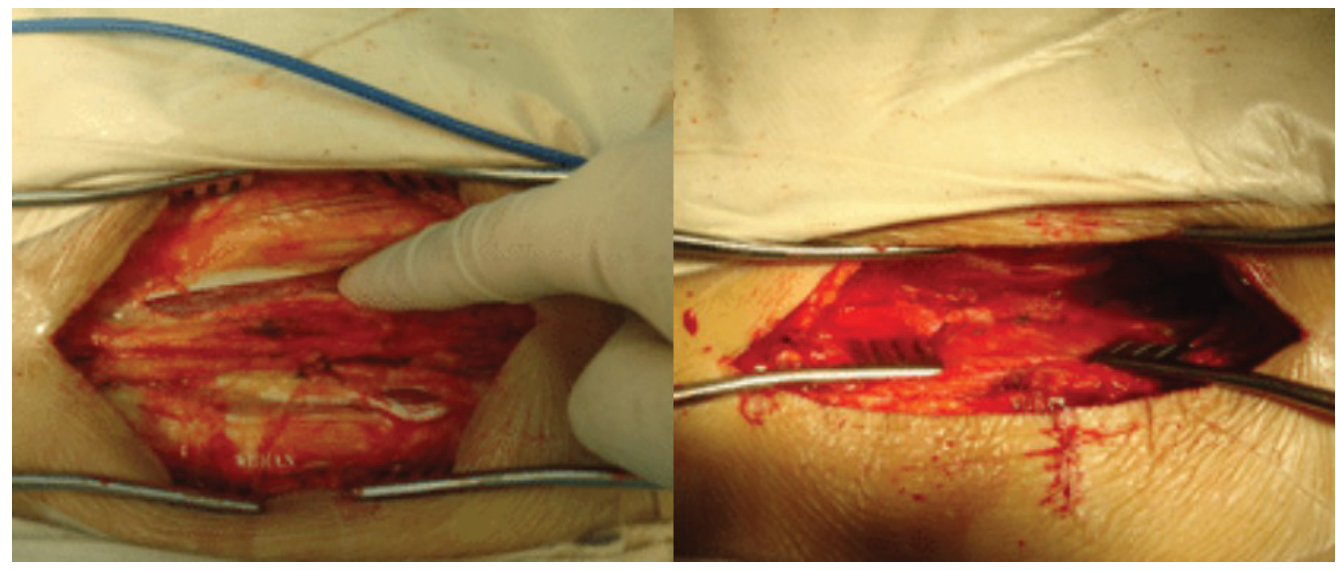

Figure 2: The picture shows the real intraoperative picture by Wiltse approach. 
Citation: Wei B, Liu T, Li H, et al. (2021) Analysis of the Advantages of Wiltse Approach Ligamentotaxis Method for Thoracolumbar Fractures. J Orthop Surg Tech 4(2):364-374

Table 4: Comparison of the Frankel results of the two groups.

\begin{tabular}{|c|c|c|c|c|c|c|}
\hline \multicolumn{7}{|c|}{ Frankel grade of Wiltse group } \\
\hline \multirow[t]{2}{*}{ Preoperative } & \multirow[b]{2}{*}{ Total } & \multicolumn{5}{|c|}{ Postoperative (12 months) } \\
\hline & & A & B & C & D & $\mathrm{E}$ \\
\hline A & 3 & 0 & & & & \\
\hline B & 5 & & 2 & & & \\
\hline C & 5 & & & 3 & & \\
\hline D & 11 & & & & 6 & \\
\hline$E$ & 28 & & & & & 41 \\
\hline Total & 52 & 0 & 2 & 3 & 6 & 41 \\
\hline \multicolumn{7}{|c|}{ Frankel grade of Traditional group } \\
\hline \multirow[t]{2}{*}{ Preoperative } & & \multicolumn{5}{|c|}{ Postoperative (12 months) } \\
\hline & Total & A & B & C & D & $E$ \\
\hline A & 2 & 0 & & & & \\
\hline B & 2 & & 0 & & & \\
\hline C & 13 & & & 2 & & \\
\hline D & 13 & & & & 9 & \\
\hline $\mathrm{E}$ & 21 & & & & & 40 \\
\hline Total & 51 & 0 & 0 & 2 & 9 & 40 \\
\hline
\end{tabular}

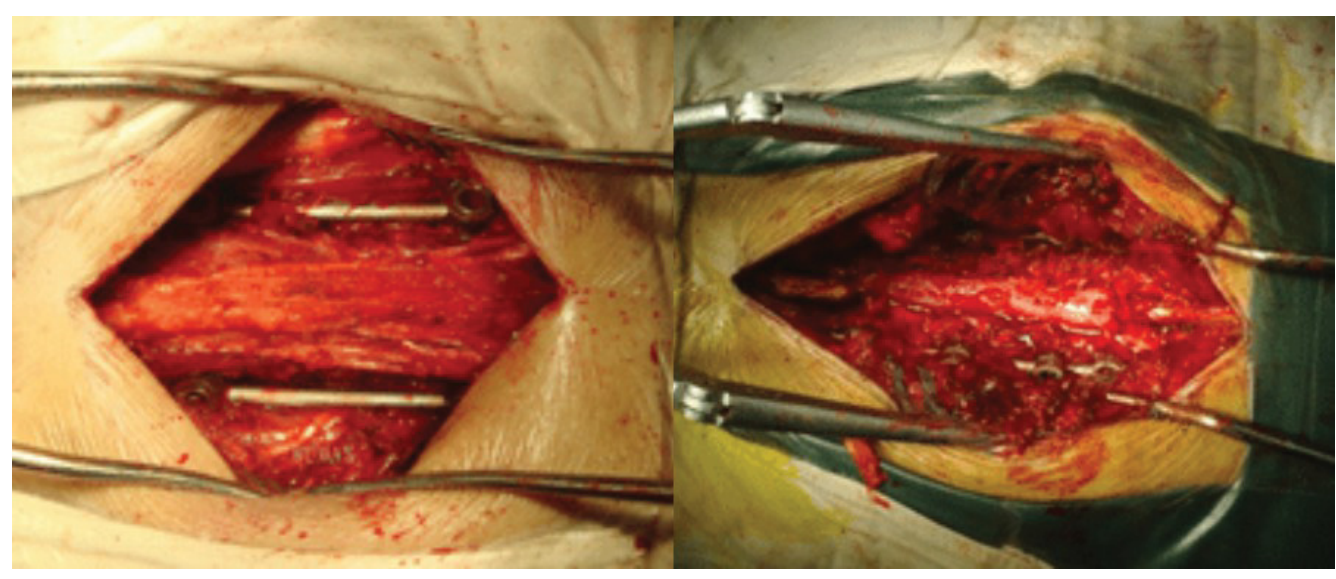

Figure 3: Intraoperative pictures showing Wiltse approach and traditional approach.

thoracolumbar spine fractures (Figure 1 and Figure 2). The advantages of the WALT compared with the traditional direct decompression approach are: (1) Less bleeding; (2) Shorter operative time; (3) The use of a natural gap approach avoids damage to the paravertebral muscle by substantial lateral pulling and stripping of the muscle and preserves the integrity of the posterior longitudinal ligament complex (Figure 3): The E.M. Laasonen study showed a significant association between postoperative lumbar pain and muscle tissue atrophy and replacement of muscle tissue by adipose tissue in the lumbar spine (Figure 4) [9]; (4) Less likely to damage the posterior branch of the lumbar nerve and the dorsal branch of the lumbar artery to prevent loss of neurogenic atrophy of the sacrospinous muscle: Liu Junhui analyzed the Wiltse approach and the posterior open approach for short-segment internal fixation of the vertebral arch. Electrophysiologically, 12 months after surgery, the mean wave amplitude of the posterior open group was significantly lower than that of the Wiltse group, and the median frequency decrease rate was significantly higher than that of the Wiltse group [10]; (5) Sufficient surgical field could be provided for pedicle screw placement; (6) Less postoperative drainage, shorter hospital stay, and faster recovery. Kyung Yun Moon, compared with the traditional direct decompression approach, the effectiveness was similar, but the former was minimally invasive Wiltse indirect decompression approach can better protect the multifidus muscle [11].

The posterior longitudinal ligament is located posterior to the vertebral body in the spinal canal, is narrow and tough, and is the long ligament of the spine, closely connected to 


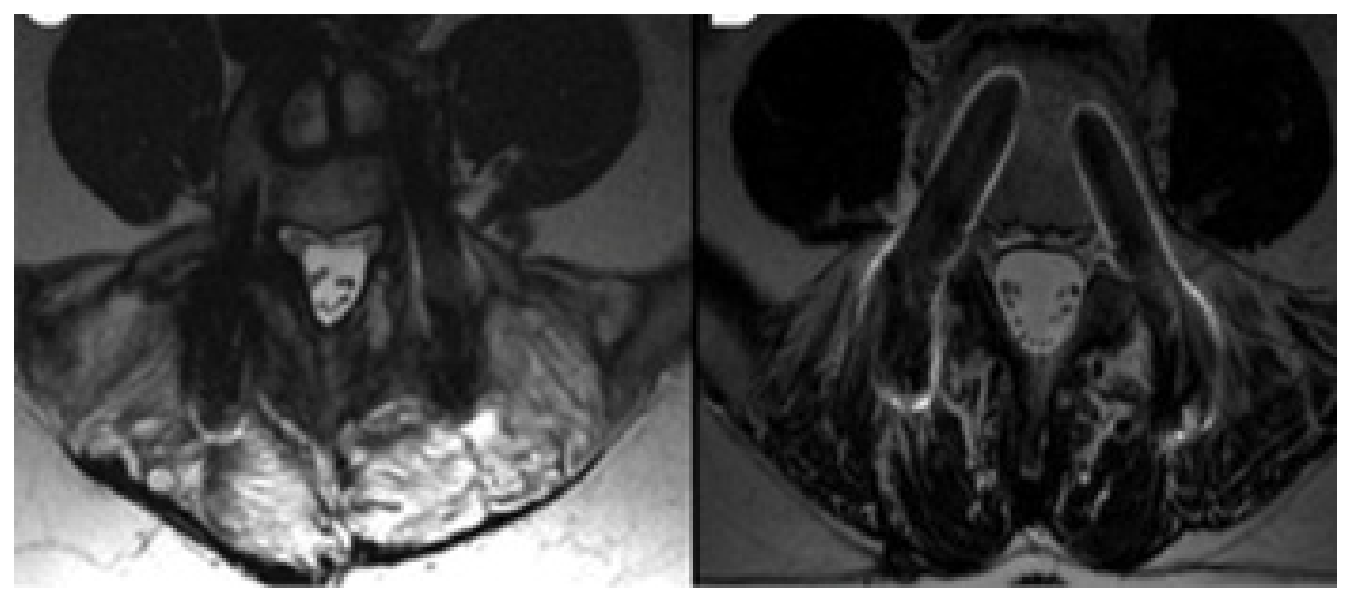

Figure 4: On the left, a T2-weighted image of the axial MRI after open lumbar fusion in the L4-L5 midline (at the level of the L5 arch), showing that the paravertebral muscles were replaced by fat; on the right, a T2-weighted image of the axial position after lumbar fusion via Wiltse approach in the same patient at L5-S1, showing that the paravertebral muscle structures were preserved.
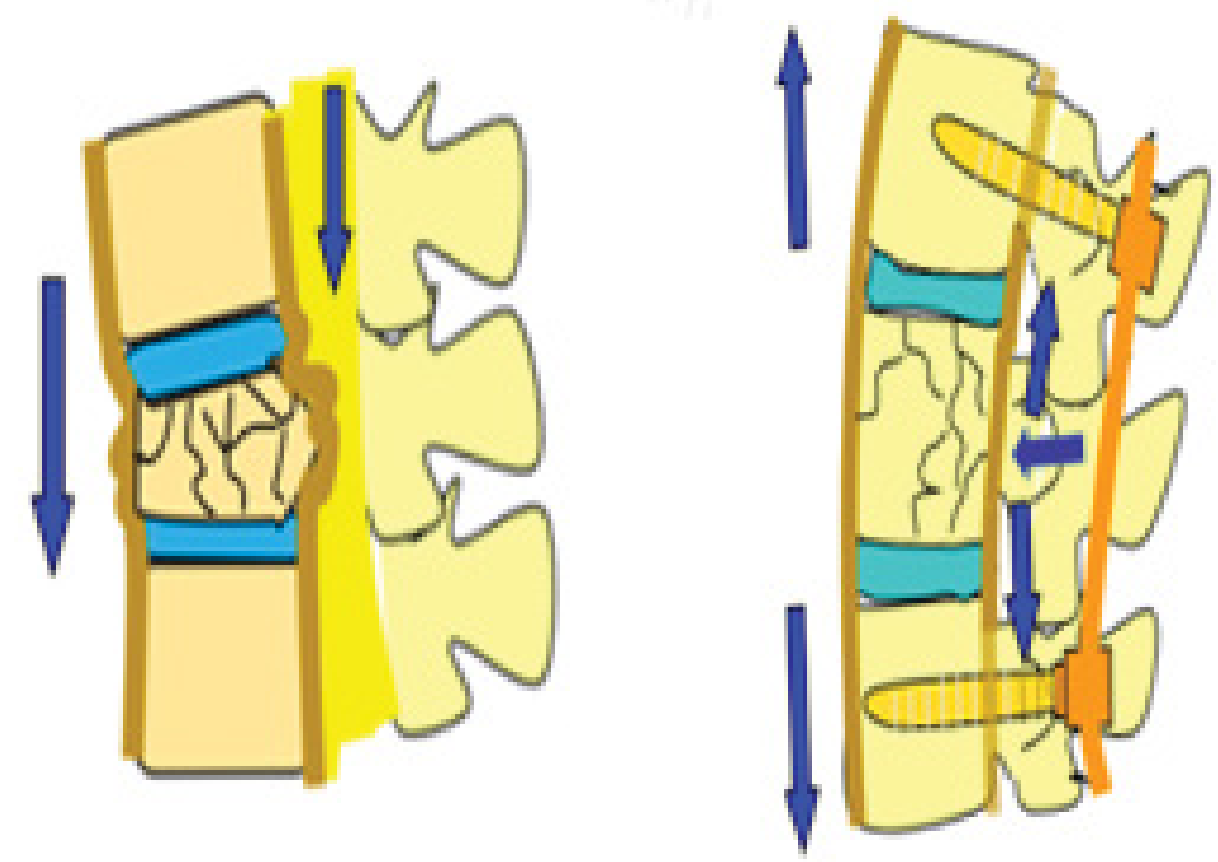

Figure 5: Schematic picture of operation.

the upper and lower margins. The criteria for assessing damage to the posterior longitudinal ligament are based on the theory proposed by Vaccaro [4] that the indirect imaging manifestations of posterior longitudinal ligament rupture in thoracolumbar fractures include intervertebral displacement or rotation. The damage of the posterior longitudinal ligament can be classified as intact, indeterminate and interrupted by preoperative X-ray, CT and MRI. We conclude that indirect decompression can be attempted in cases where the posterior longitudinal ligament is assessed as "intact" or "indeterminate" on preoperative imaging, either through a median approach or a Wiltse approach. In attempting to reposition the fracture through posterior longitudinal ligament tension, a system of longitudinal bracing on both sides of the arch nail is inserted, creating tension on the posterior longitudinal ligament and thus indirectly repositioning the fracture (Figure 5). This approach generally allows for anatomic repositioning of the bone in the spinal canal without direct decompression. In "indeterminate" cases, indirect decompression can be attempted with the Wiltse approach. If the fracture cannot be repositioned, then it is confirmed that there is a posterior longitudinal ligament injury that does not produce the necessary tension to reposition the fracture, in which case a partial laminectomy with direct decompression is required. In this study, we also confirmed that indirect decompression along the Wiltse approach is possible even in the presence of severe occupancy of the spinal canal and neurological impairment, thus achieving the same neurological recovery and repositioning results as in the traditional direct decompression approach group. In 


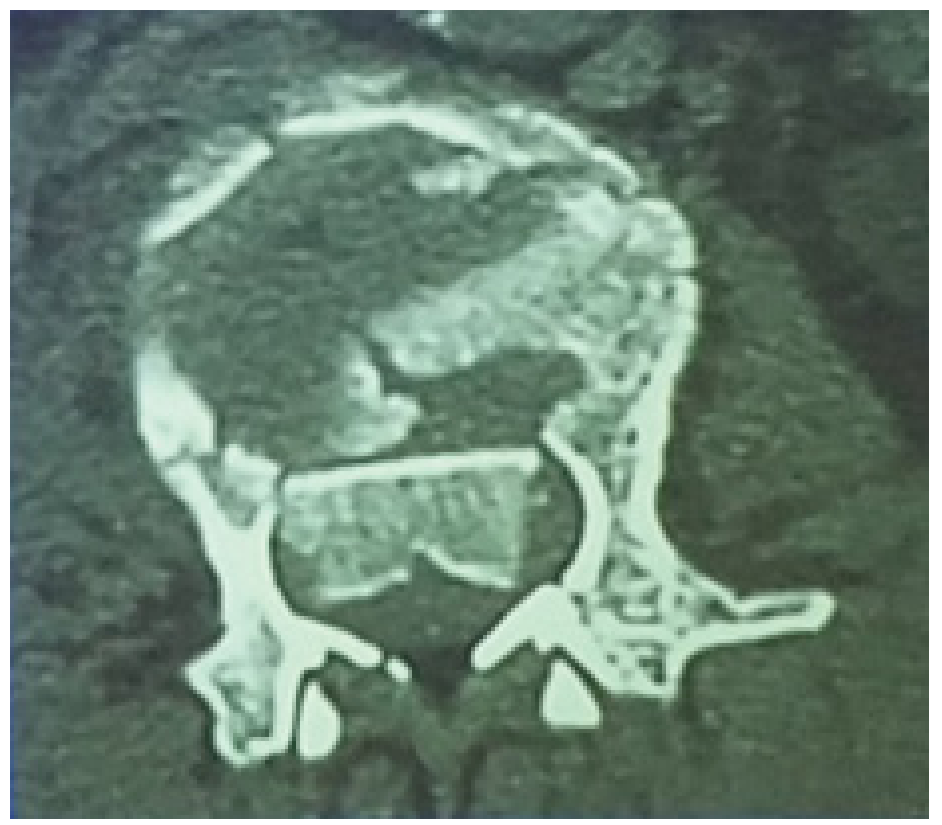

Figure 6: Cases in which the ligament tension repositioning method was not applicable, when there was reversal of the fracture fragment then it indicated that the posterior longitudinal ligament was damaged and indirect repositioning could not be performed and was not included in the study.

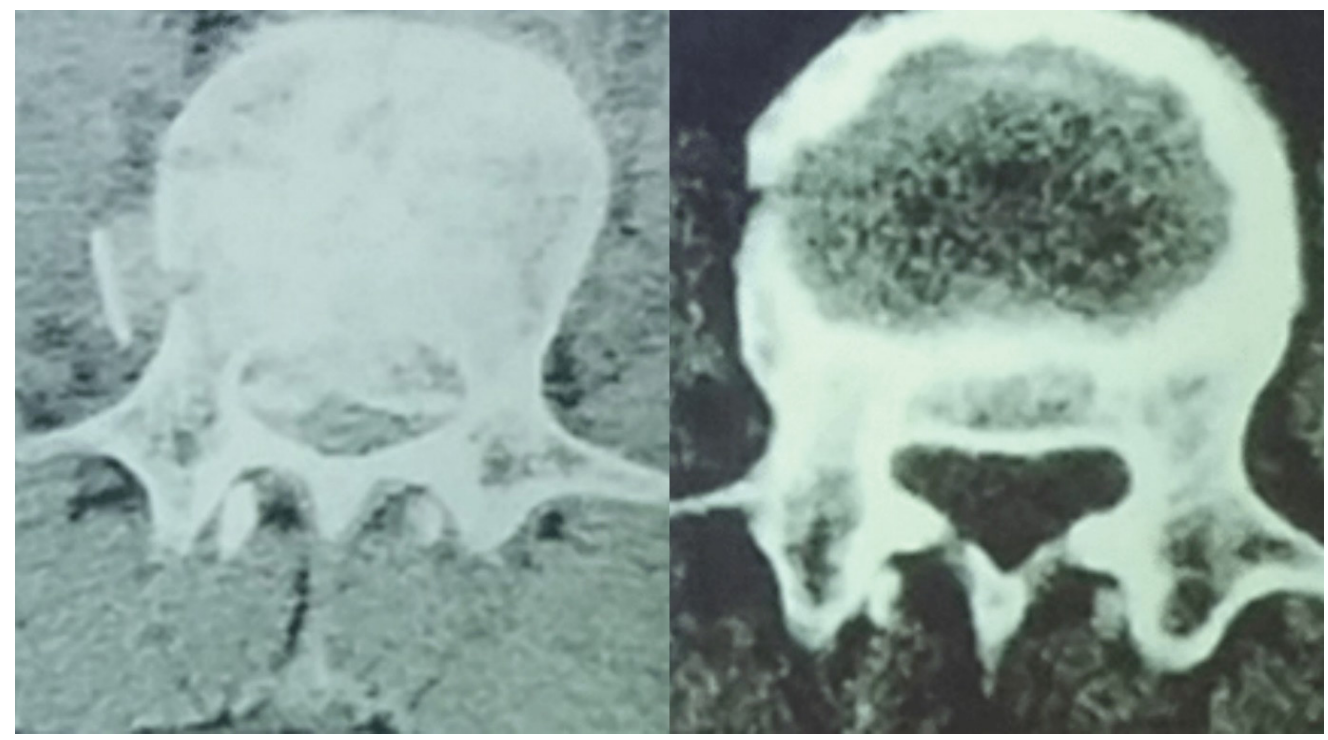

Figure 7: Reshaping and reconstruction of the spinal canal at 12 months post-injury, with an increase in canal diameter from $50 \%$ to $75 \%$ of normal, as shown in the figure.

addition, preoperative examination is also a sign of rupture of the posterior longitudinal ligament complex if there is a large spinous process gap or a serious change in the relative position of the two upper and lower spinous processes [4]. In this case, direct decompression is preferable.

In addition, the phenomenon in which the fragments of the posterior wall of the vertebral body are usually turned $180^{\circ}$, with the osteophytic surface of the fragment facing posteriorly in the spinal canal and the cortical surface of the bone (posterior wall) facing anteriorly, is known as fracture block overturning, and this is generally considered to be evidence of posterior longitudinal ligament rupture, and direct reduction by tension of the posterior longitudinal ligament is ruled out (Figure 6). However, Arlet [12], after sagittal CT reconstruction of patients with thoracolumbar burst fractures, found a "pseudo-reversed cortical sign", a portion of the imaging resembling a reversed fracture mass that is actually a portion of the upper or lower endplate being compressed into the crushed vertebral body, in which case the posterior longitudinal ligament is continuous and could theoretically. In this case, the posterior longitudinal ligament is continuous and can theoretically be indirectly repositioned by tension decompression of the posterior longitudinal ligament. The key to distinguishing between fracture mass 
reversal and pseudo-reverse cortical sign is to scan as many levels of the injured vertebrae as possible in the axial position to facilitate observation during CT examination.

The bone mass in the spinal canal can be repositioned by ligamentous tension, thus allowing avoidance of incisional decompression. Moreover, even without decompression, the bone mass in the spinal canal can be gradually reduced or resorbed by biomechanical action (Figure 7). This idea has been reported in several publications [13,14]. Vaccaro [15] suggested that stenosis is reduced by more than $50 \%$ through spinal canal remodeling, regardless of surgical or conservative treatment, because the fragmented bone masses in the spinal canal are slowly absorbed during biomechanically stable remodeling of the spine, as shown in Figure 8, Wilcox RK designed an experiment to show by CT whether spinal canal volume is associated with nerve injury correlation. It was shown that burst fracture is a dynamic process in which the spinal canal pressure peaks during impingement, and there was no significant correlation between the degree of posterior canal occlusion seen on CT and the maximum amount of occlusion that occurred at impingement [16]. Johnsson R [17] showed that even without surgery, the fracture mass in the spinal canal is cleared by gradual remodeling, resulting in surgical decompression. Qiu [18] used a finite element model to analyze the mechanism of injury in T12-L1 burst fractures and found that the canal stenosis was less at the most advanced stage of impact than at the previous peak. These findings could explain the minimal relationship between clinically observed neurological deficits and traumatic spinal stenosis. Yang [19] demonstrated that the diameter of the spinal canal could be immediately restored by indirect reduction using a short segmental pedicle nailing system without fusion. The above literature confirms that either indirect or direct decompression is not necessarily sought to achieve complete recovery of the intracanal volume after surgery, as it does not correlate well with the final recovery of neurological function and spinal stability.

Combining the Wiltse approach with the posterior longitudinal ligament tension repositioning method, WALT allows ideal repositioning of most thoracolumbar vertebral fractures, thus achieving indirect decompression, avoiding both the stripping of the paravertebral muscle and the removal of the vertebral plate and other structures, thus avoiding the medical source injury of spinal canal decompression, and can significantly reduce the It can significantly reduce complications such as degeneration of the paravertebral muscles and the incidence of postoperative low back pain, reduce intraoperative and postoperative bleeding, and reduce

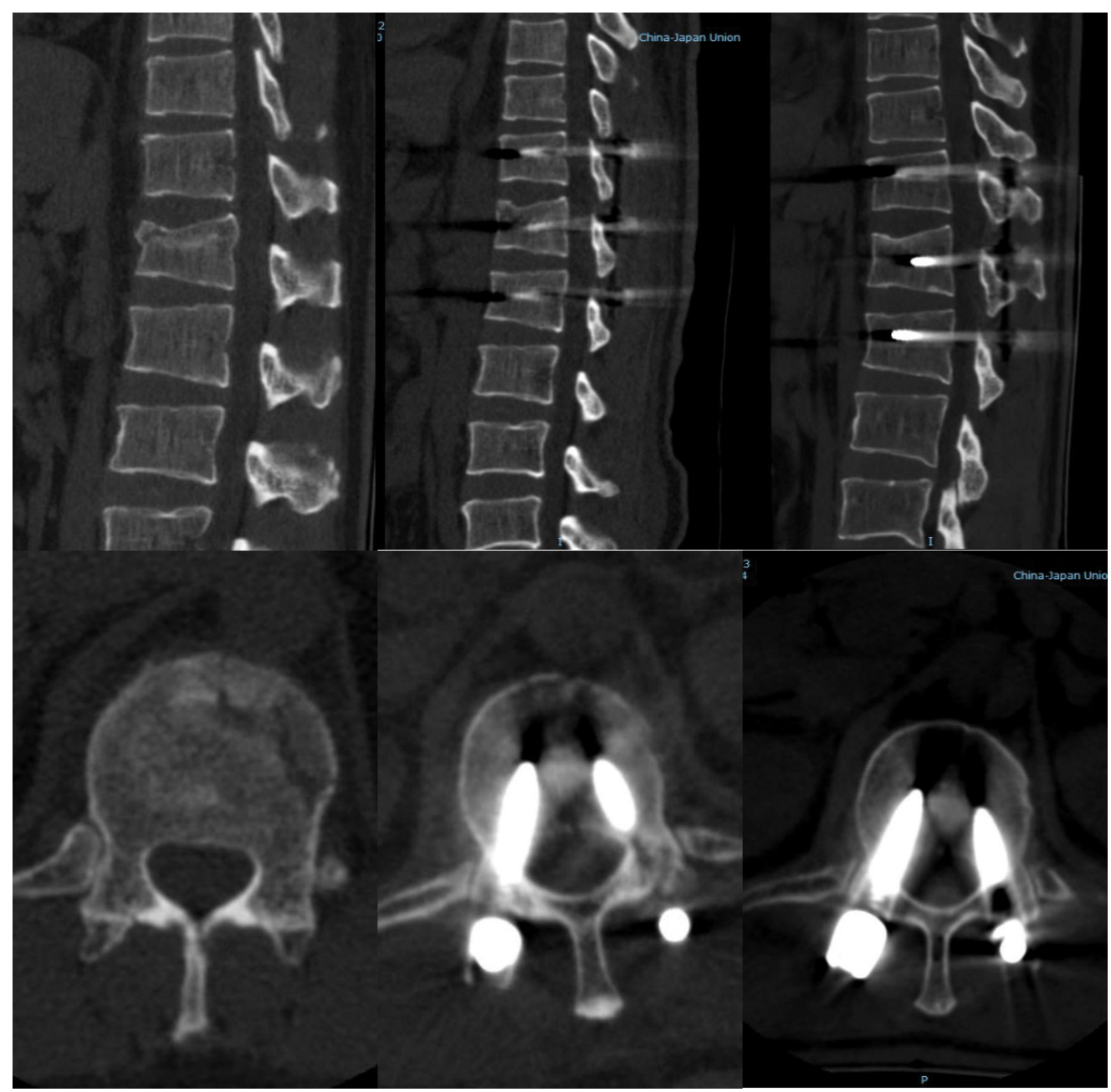

Figure 8: Pre-operative, immediate post-operative and 1 year post-operative access via the paravertebral space. 
hospitalization time and costs. It can be used in most cases of thoracolumbar fractures with intact posterior longitudinal ligaments, and the clinical effectiveness is consistent with that of traditional surgery, and it has many advantages and is simple to operate.

The limitations of this retrospective study are: the followup period was too short, only 1 year, due to difficulties in case counting, and more valuable conclusions could be obtained if long-term follow-up was performed; and if there were enough cases, further group comparisons could be made, i.e., the median approach direct decompression group, median approach indirect decompression group, and Wiltse approach indirect decompression group, which could yield more refined and further results. This will provide us with more refined and further results. This also provides insight for our subsequent study.

\section{Conclusion}

The WALT for thoracolumbar fractures has many advantages over the traditional direct decompression reduction internal fixation approach: less bleeding, shorter operative time, no muscle stripping, less postoperative and long-term follow-up pain, less postoperative drainage, shorter hospital stay, and less expense. There is no significant difference between the two repositioning effects in terms of Cobb angle change and vertebral body height recovery. While the traditional concept is that tension posterior longitudinal ligament repositioning is less effective than direct decompression with removal of the lamina in patients with neurological impairment in thoracolumbar fractures, the findings of this study confirm that there is no difference in long-term recovery of neurological function between indirect decompression and direct decompression when the posterior longitudinal ligament is intact in patients with neurological impairment in thoracolumbar fractures. Therefore, for patients with thoracolumbar fractures in which the posterior longitudinal ligament is unbroken or the posterior ligamentous complex is intact, regardless of whether they have neurological impairment, the WALT is more advantageous in clinical practice.

\section{Availability of Data and Materials}

Not applicable.

\section{Acknowledgements}

None.

\section{Funding}

This work was supported by the Department of Science and Technology of Jilin Province (project number: 20200404187YY).

\section{Ethics Declarations}

\section{Ethics approval and consent to participate}

All procedures performed in studies involving human participants were in accordance with the ethical standards of the institutional and/or national research committee and with the 1964 Helsinki Declaration and its later amendments or comparable ethical standards.

\section{Consent for publication}

Not applicable.

\section{Competing interests}

The authors declare that they have no competing interests.

\section{References}

1. Wood KB, Li W, Leb DR, et al. (2014) Management of thoracolumbar spine fractures. Spine J 14: 145-164.

2. Walker CT, Xu DS, Godzik J, et al. (2018) Minimally invasive surgery for thoracolumbar spinal trauma. Ann Transl Med 6: 102.

3. Rantanen J, Hurme M, Falck B, et al. (1993) The lumbar multifidus muscle five years after surgery for a lumbar intervertebral disc herniation. Spine 18: 568-574.

4. Vaccaro AR, Lehman RA, Hurlbert RJ, et al. (2005) A new classification of thoracolumbar injuries: The importance of injury morphology, the integrity of the posterior ligamentous complex, and neurologic status. Spine 30: 2325-2333.

5. Vu C, Gendelberg D (2020) Classifications in brief: AO thoracolumbar classification system. Clin Orthop Relat Res 478: 434-440.

6. Reinhold M, Knop C, Beisse R, et al. (2010) Operative treatment of 733 patients with acute thoracolumbar spinal injuries: Comprehensive results from the second, prospective, Internetbased multicenter study of the spine study group of the German association of trauma surgery. Eur Spine J 19: 1657-1676.

7. Wiltse LL, Bateman JG, Hutchinson RH, et al. (1968) The paraspinal sacrospinalis-splitting approach to the lumbar spine. J Bone Joint Surg Am 50: 919-926.

8. Jiang R, Wu H, Wang JC, et al. (2011) Paraspinal approach for thoracolumbar fracture. Chin J Traumatol 14: 3-6.

9. Laasonen EM (1984) Atrophy of sacrospinal muscle groups in patients with chronic, diffusely radiating lumbar back pain. Neuroradiology 26: 9-13.

10. Junhui $L$, Zhengbao $P$, Wenbin $X$, et al. (2017) Comparison of pedicle fixation by the Wiltse approach and the conventional posterior open approach for thoracolumbar fractures, using MRI, histological and electrophysiological analyses of the multifidus muscle. Eur Spine J 26: 1506-1514.

11. Moon KY, Lee SE, Kim KJ, et al. (2013) Back muscle changes after pedicle based dynamic stabilization. J Korean Neurosurg Soc 53: 174-179.

12. Arlet V, Orndorff DG, Jagannathan J, et al. (2009) Reverse and pseudoreverse cortical sign in thoracolumbar burst fracture: Radiologic description and distinction-a propos of three cases. Eur Spine J 18: 282-287.

13. Jeong WJ, Kim JW, Seo DK, et al. (2013) Efficiency of ligamentotaxis using PLL for thoracic and lumbar burst fractures in the load-sharing classification. Orthopedics 36: e567-574.

14. Wang XB, Lü GH, Li J, et al. (2017) Posterior distraction and instrumentation cannot always reduce displaced and rotated posterosuperior fracture fragments in thoracolumbar burst fracture. Clin Spine Surg 30: E317-E322.

15. Vaccaro AR, Kim DH, Brodke DS, et al. (2004) Diagnosis and 
management of thoracolumbar spine fractures. Instr Course Lect 53: 359-373.

16. Cieślik P, Floriańczyk A, Kwiatkowski K, et al. (2013) Thoracolumbar compression fractures - experimental study and clinical case analysis. Ortop Traumatol Rehabil 15: 139-147.

17. Johnsson R, Herrlin K, Hägglund G, et al. (1991) Spinal canal remodeling after thoracolumbar fractures with intraspinal bone fragments. 17 cases followed 1-4 years. Acta Orthop Scand 62: 125-127.
18. Qiu TX, Tan KW, Lee VS, et al. (2006) Investigation of thoracolumbar T12-L1 burst fracture mechanism using finite element method. Med Eng Phys 28: 656-664.

19. Yang HL, Shi JH, Liu J, et al. (2009) Fluoroscopically-guided indirect posterior reduction and fixation of thoracolumbar burst fractures without fusion. Int Orthop 33: 1329-1334.

DOI: $10.36959 / 453 / 574$

Copyright: (C) 2021 Wei B, et al. This is an open-access article distributed under the terms of the Creative Commons Attribution License, which permits unrestricted use, distribution, and reproduction in any medium, provided the original author and source are credited. 